\section{P43 FACTORS INFLUENCING LENGTH OF CHEST DRAIN INSERTION IN CHILDREN WITH EMPYEMA}

M Osinibi, C Sun, C Bossley, G Ruiz, R Abusamra. King's College Hospital, London, UK

10.1136/thoraxjnl-2017-210983.185

Introduction Many factors might influence length of chest drainage (LOCD) when managing parapneumonic effusions/ empyema including effusion size, septations, extent of pneumonia, presence of air leaks, pathogen virulence, fibrinolytic therapy with urokinase and local policies. UK guidelines recommend using the same fixed volumes for infants and for all children older than a year regardless of size. This preliminary retrospective survey was undertaken to determine whether there may be a signal to suggest an optimal urokinase volume based on weight which might warrant a controlled study.

Objectives To investigate clinical factors that affect length of chest drain insertion (LOCD) in children with empyema and to examine if there is an optimal urokinase dosing based on patient's weight would affect the LOCD.

Methods We conducted a retrospective review of clinical data from 52 children with empyema admitted to our centre between January 2015-December 2016. Chest drains were in place for a range of 2 to 12 days with a median of 5 days. We conducted a comparison of these data between the group of patients who required chest drain insertion for $\leq 5$ days and those $\geq 5$ days. We also grouped the patients into two levels of urokinase dose based on patients weight to create a frequency table and eventually to look if a certain dose is associated with a shorter LOCD.

Results The median LOCD insertion in our group was 5 days. Patients with a longer LOCD showed a trend to be younger and had a higher WCC, but this was not statistically significant. There were no statistically significant difference in the dose $/ \mathrm{kg}$ of urokinase and LOCD.

Conclusion Our study did not show weather a certain urokinase dose based on weight would affect the LOCD. There were no other clinical indicators among our population that can predict the LOCD.

\begin{tabular}{llll} 
Abstract P43 Table 1 & & & \\
\hline & LOCD $\leq 5$ days & LOCD $>5$ days & P \\
& n. (28) & n.(22) & value \\
\hline Gender (Male) & 12 & 11 & 0.77 \\
Age (Mean) & 5.36 & 2.90 & 0.38 \\
O2 requirement & 19 & 17 & 0.58 \\
CRP & $231(43-346)$ & $241(96-440)$ & 0.54 \\
WCC & $16.35(0-59)$ & $20.18(7.99-$ & 0.22 \\
& & $96.09)$ & \\
US fluid depth (mm) & $34.6(18.9-$ & $30(16-74)$ & 0.37 \\
& $85)$ & & \\
Urokinase U/Kg & $2278(404-$ & $2667(317.5-$ & 0.42 \\
& $3922)$ & $5405)$ & \\
Frequency of patients on low dose & 9 & 9 & 0.76 \\
Urokinase (<2000 u/kg) & & & \\
Frequency of patients on high dose & 18 & 13 & 0.76 \\
Urokinase ( $\geq 2000 \mathrm{u} / \mathrm{kg})$ & & & \\
\hline
\end{tabular}

\section{P44 THE UTILITY OF BEDSIDE LUNG ULTRASOUND IN THE ASSESSMENT OF EMERGENCY MEDICAL ADMISSIONS PRESENTING WITH ACUTE DYSPNOEA: A PROSPECTIVE ANALYSIS}

${ }^{1}$ B Chakrabarti, ${ }^{1} \mathrm{C}$ Curtis, ${ }^{1} \mathrm{~A}$ Kwok, ${ }^{2} \mathrm{M}$ Gautam. ${ }^{1}$ University Hospital Aintree, Liverpool, UK; ${ }^{2}$ Royal Liverpool University Hospital, Liverpool, UK

10.1136/thoraxjnl-2017-210983.186

Introduction Lung Ultrasound (LUS) is emerging as a potentially useful bedside investigation to aid assessment of acute dyspnoea. However, little UK based data exists regarding the impact of LUS when performed over and above standard care in subjects presenting to hospital with acute dyspnoea.

Methodology Subjects presenting with acute dyspnoea as Emergency Admissions to 2 University Hospitals were evaluated within 24 hours of admission by a Respiratory Physician with Level 2 Ultrasound competency. A chest radiograph and appropriate blood tests had been performed beforehand. In all cases, the precise aetiology of the subject's dyspnoea remained uncertain following review of the patient/existing investigations by the Respiratory Physician but prior to bedside LUS being performed.

Results 80 subjects (Age 68 (SD 17) years; 43\% Male) were included with 77 surviving to discharge. LUS findings comprised Diffuse Bilateral B lines suggestive of Acute Heart Failure (AHF)/Interstitial Syndrome in 29\% (23/80), Consolidation in $23 \%(18 / 80)$, A lines/"normal" in $24 \%(19 / 80)$, unilateral focal B lines in 21\% (17/80), unspecified sub-pleural focus (1/ 80 ), small pleural effusion (1/80) and loss of lung sliding/lung point indicative of pneumothorax (1/80). In the 18 cases of consolidation and the 23 cases of AHF identified on LUS, only 39\% (7/18) and 30\% (7/23) of the plain chest radiographs respectively were subsequently reported by a radiologist as showing any parenchymal abnormalities. In 29\% (23/80) cases, the addition of LUS was felt to have given a specifically alternative diagnosis to the patient's presentation not previously considered (7/23 Pneumonia, 12/23 AHF, 2/23 Abdominal Sepsis, 1 Pneumothorax, 1 Pulmonary embolism) whilst in a further $44 \%(35 / 80)$ cases, LUS was felt to have strengthened the certainty of an existing differential diagnosis considered prior to LUS being performed. The diagnosis made post LUS was found to be concordant with the subject's "Discharge Diagnosis" in $83 \%(66 / 77)$ cases.

Conclusion Use of bedside LUS as an adjunct to standard care in the assessment of acute dyspnoea often resulted in either the consideration of an alternative diagnosis to account for a patient's symptoms or offered clinicians greater certainty on an existing differential diagnosis thus better directing management.

\section{Cellular insights into lung injury repair}

\section{P45 MECHANISMS OF REGENERATION: RETINOIC ACID ACTS VIA THE ENDOTHELIUM TO DRIVE HUMAN LUNG REPAIR}

J Alçada, DS Shao, MJD Griffiths, CH Dean, M Hind. National Heart and Lung Institute, Imperial College London, London, UK

10.1136/thoraxjnl-2017-210983.187 
A
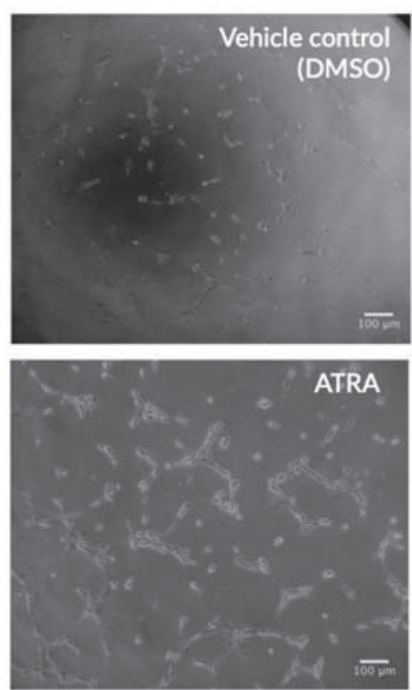

B

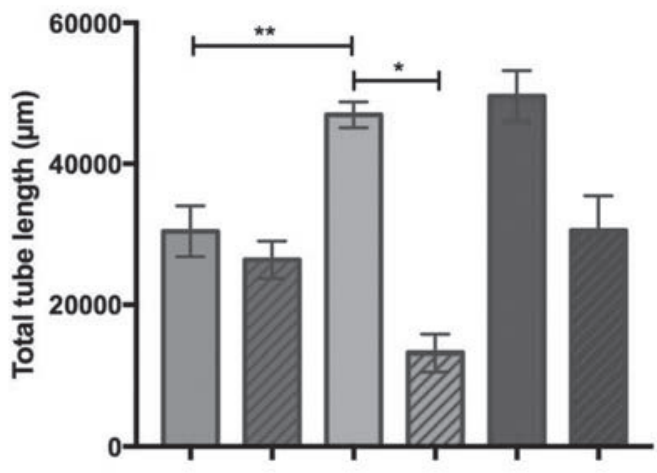

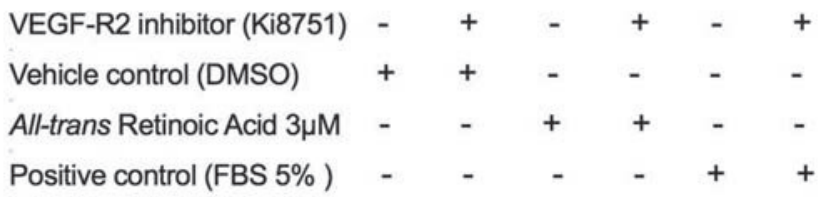

Abstract P45 Figure 1 ATRA induces pulmonary microvascular angiogenesis through VECF-Receptor 2. HPMECs were seeded onto matrigel and tube formation assessed after 4 hours. Representative images shown, original magnification x10 (A). ATRA $3 \mu \mathrm{M}$ significantly increased total tube formation when compared to vehicle control (DMSO 0.03\%) and this effect was abolished by the selective VEGF-Receptor 2 inhibitor Ki8751 (Tocris) (B). (Results presented as Mean $\pm S E M,{ }^{*} p<0.05$ Mann-Whitney test, $n=6$ (2 independent experiments with 3 replicates per condition).

Retinoic acid (RA) is essential for correct mammalian lung development and remarkably can induce alveolar regeneration in rodent models of COPD. However, clinical trials of RA and RA-Receptor gamma agonists in patients with COPD were disappointing. The reasons for the lack of effect in COPD patients are unknown but there is a clear need for a greater understanding of the underlying biology driving RA-induced lung regeneration. To investigate this, I studied the role of RA in human alveolar repair using isolated human pulmonary microvascular endothelial cells (HPMEC), alveolar epithelial cells (A549) and developed a novel human alveolar model using Precision-Cut Lung Slices (PCLS). In HPMECs, All-trans RA (ATRA) induced angiogenesis in a dose dependent manner $(\mathrm{p} \leq 0.01, \mathrm{n}=5)$. Pharmacological inhibition of VEGF receptor-2 with the selective inhibitor Ki8751 (Tocris) abolished this effect $(\mathrm{p} \leq 0.05, \mathrm{n}=6)$ (figure 1$)$. A proteome profiler array of PCLS, demonstrates an increase in pro-angiogenic proteins in the ATRA group, including CXCL16, IGFBP-3, PIGF, VEGFA, HB-EGF and MCP-1. In addition, ATRA treatment of PCLS generated from histologically normal human lung lead to increased endothelial (PECAM-1) and alveolar type 2 (ProSPC) cell markers $(n=3)$. Further investigation revealed that in wound-healing (scratch) assays of confluent cell monolayers, ATRA had no direct effect on the rate of wound healing in alveolar epithelial cells (A549) but significantly increased healing in HPMECs $(\mathrm{p} \leq 0.01, \mathrm{n}=3)$. Moreover, siRNA knockdown of VEGF-R2 inhibited ATRA-induced wound-healing in HPMECs. Conditioned media from ATRA-treated HPMEC increased wound healing in A549 cells suggesting that the effects of RA on alveolar epithelial repair are mediated indirectly via the vascular network. HPMEC secreted HB-EGF with ATRA stimulation and HB-EGF treatment significantly increased A549 wound healing $(n=2)$ suggesting it may act as a paracrine endothelial-epithelial regulator. My work demonstrates that RA has biological activity in human lung with direct effects on human lung microvasculature including cell migration, angiogenesis, and regulation of proteins likely to be important in alveolar repair. Together my data significantly advances our understanding of the mechanisms of RA induced repair in human lung tissue.

\section{P46 CIGARETTE SMOKE- AND HYPOXIA-INDUCED IMBALANCED VASOACTIVE GENE EXPRESSION IN HUMAN PULMONARY ARTERY ENDOTHELIAL AND SMOOTH MUSCLE CELLS}

A Alqarni, O Brand, A Pasini, M Alshehri, L Pang. Division of Respiratory Medicine, School of Medicine, University of Nottingham (City Hospital), Nottingham, UK

\subsection{6/thoraxjnl-2017-210983.188}

Background Pulmonary Hypertension (PH) is a common and serious complication of Chronic Obstructive Pulmonary Disease (COPD) associated with increased mortality and morbidity and characterised by Pulmonary Artery Smooth Muscle Cell (PASMC) hyperproliferation and vascular remodelling. Studies suggest that chronic hypoxia and Cigarette Smoke (CS) can cause aberrant PASMC proliferation and vascular remodelling, however, how cigarette smoke and hypoxia contribute to pulmonary artery wall thickening and $\mathrm{PH}$ in COPD is not fully understood. We hypothesise that hypoxia and CS can induce an imbalance between excessive vasoconstrictors and deficient vasodilators, which then contribute to aberrant PASMC proliferation in COPD-associated $\mathrm{PH}$ and can be a target for therapeutic intervention.

Method To prove the hypothesis, confluent Human Pulmonary Artery Smooth Muscle Cells (hPASMCs) and Human Pulmonary Artery Endothelial Cells (hPAECs) were treated with different concentrations of Cigarette Smoke Extract (CSE) (1\%, $2.5 \%$, and $5 \%)$ under normoxic $\left(21 \% \mathrm{O}_{2}\right)$ or hypoxic $(1 \%$ $\mathrm{O}_{2}$ ) condition for 72 hour. The protein and mRNA expression of Prostacyclin Synthase (PGIS), Cyclooxygenase-2 (COX-2), Endothelial Nitric Oxide Synthase (eNOS), Thromboxane A Synthase (TXAS), and Endothelin 1(ET-1) was analysed by Western blotting and real-time RT-PCR, respectively.

Results The expression of vasodilator genes eNOS and PGIS was noticeably downregulated in both hPASMCs and hPAECs, 